\title{
Fast track and outpatient surgery in total knee arthroplasty: beneficial for patients, doctors and hospitals
}

\author{
Michael T. Hirschmann ${ }^{1,2} \cdot$ Nanne Kort $^{4} \cdot$ Sebastian Kopf $^{3} \cdot$ Roland Becker $^{3}$
}

Received: 21 July 2017 / Accepted: 25 July 2017 / Published online: 22 August 2017

(C) European Society of Sports Traumatology, Knee Surgery, Arthroscopy (ESSKA) 2017

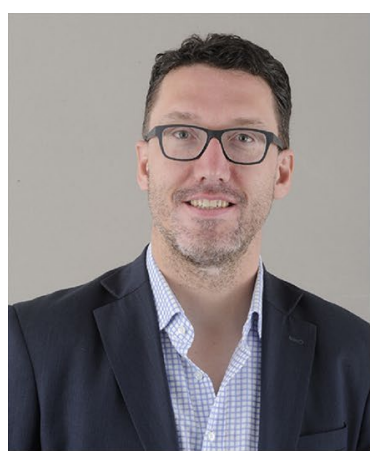

Michael T. Hirschmann

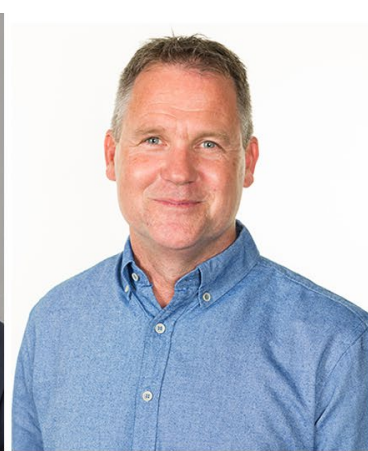

Nanne Kort

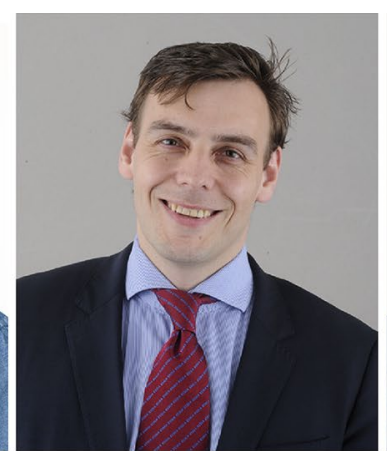

Sebastian Kopf

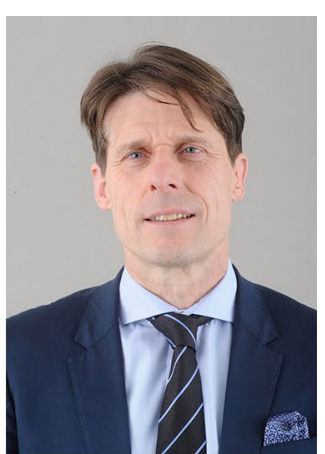

Roland Becker
These days many knee surgeons talk about fast track (FT) or outpatient pathways in total knee arthroplasty (TKA). It might be due to the increasingly difficult economic health care climate coming along with increasing financial pressure and decreasing reimbursement that FT knee arthroplasty is one of the hot topics.

Michael T. Hirschmann

michael.hirschmann@ksbl.ch; michael.hirschmann@unibas.ch; Michael_Hirschmann@web.de

http://www.kneedoctor.ch

1 Department of Orthopaedic Surgery and Traumatology, Kantonsspital Baselland (Bruderholz, Liestal, Laufen), 4101 Bruderholz, Switzerland

2 University of Basel, 4000 Basel, Switzerland

3 Department of Orthopedics and Traumatology, Centre of Joint Replacement, Hospital Brandenburg and "Theodor Fontane" Medical School, 14770 Brandenburg/Havel, Germany

4 Department of Orthopedics and Traumatology, Zuyderland Medical Center, Sittard-Geleen, The Netherlands
Fast track programmes aim for optimising perioperative management using a multidisciplinary and individualised patient approach [5, 6]. A FT programme optimises patient care and allows an earlier discharge from hospital [5]. Outpatient surgical pathways even go one step further and aim for same day admission and discharge of the patient undergoing partial or even total knee arthroplasty $[1,2]$. Over the last 15 years, FT programmes have proven beneficial for the patient, the knee surgeon as well as the health insurance system. Reduced length of stay and decreased morbidity comes along with similar rates of readmission or adverse events [5].

In fact, the length of hospital stay for knee arthroplasty patients has significantly decreased through the last decade, but still varies widely between hospitals and countries. However, in most of the hospitals a length of stay for UKA between 1 and 5 days and for TKA between 2 and 6 days is already reality. The length of hospital stay depends on a variety of different factors such as patient characteristics, comorbidities, hospital environment, the doctors will to work in a multidisciplinary approach and finally the health care reimbursement system [5]. Clearly, the reimbursement system is 
a given variable, but with introduction of diagnosis-related groups (DRG) systems in several countries across Europe early discharge from hospital has been put into the focus of many hospital administrators.

The time of being an inpatient is one aspect, but patients' recovery time in general is another important issue. Optimising patients' management prior, during and after TKA surgery significantly, improves patients satisfaction.

The pertinent and still open question is if FT TKA is feasible in all or only well-selected groups of patients. What is the optimal patient for such a rapid recovery programme?

In a review article included in this issue, Kort et al. [4] found nine clinical studies dealing with outpatient knee arthroplasty. Low complication and readmission rates were found [4]. Clearly, the role of proper patient selection for prevention of adverse events and readmissions is highlighted [4]. In this article, Kort et al. [4] present a catalogue of expert opinion and literature-based patient selection criteria for outpatient knee arthroplasty.

In another landmark article, Kort et al. [3] compared the results of their first 20 outpatient unicondylar knee arthroplasty (UKA) patients with a conventional FT control group. Seventeen patients $(85 \%)$ in the outpatient pathway were discharged on the day of surgery as scheduled, whereas in the FT pathway $95 \%$ of the patients were discharged $<3$ days post-operatively [3]. Level of pain appeared to be the most important risk factor for prolonged stay in hospital. This might explain the fact that an adequate multimodal pain management is one decisive cornerstone of multidisciplinary rapid recovery protocols [7].

An increasing number of knee surgeons have already introduced or plan to introduce rapid recovery pathways for the care of their knee arthroplasty patients. It is not only one step, but several steps forming a whole process to change from conventional to FT knee arthroplasty. This change in medical and organisational mindset should be led by a multidisciplinary steering group of knee surgeons, anaesthetists, physician assistant, care manager, nurses, pain specialists and physiotherapists.
However, the current evidence for FT programmes in knee arthroplasty is still scarce and further research needs to be done to create: (1) proper patient selection criteria, (2) define readmission and adverse event rates in this selected cohort.

\section{Compliance with ethical standards}

Conflict of Interest The authors declare that they have no conflict of interest.

\section{References}

1. Bovonratwet P, Ondeck NT, Nelson SJ, Cui JJ, Webb ML, Grauer JN (2017) Comparison of outpatient versus inpatient total knee arthroplasty: an ACS-NSQIP analysis. J Arthroplasty 32(6): 1773-1778

2. Gromov K, Kjaersgaard-Andersen P, Revald P, Kehlet H, Husted $\mathrm{H}$ (2017) Feasibility of outpatient total hip and knee arthroplasty in unselected patients. Acta Orthop. doi:10.1080/17453674.2017. 1314158

3. Kort NP, Bemelmans YF, Schotanus MG (2015) Outpatient surgery for unicompartmental knee arthroplasty is effective and safe. Knee Surg Sports Traumatol Arthrosc. doi:10.1007/ s00167-015-3680-y

4. Kort NP, Bemelmans YF, van der Kuy PH, Jansen J, Schotanus MG (2016) Patient selection criteria for outpatient joint arthroplasty. Knee Surg Sports Traumatol Arthros. doi:10.1007/ s00167-016-4140-z

5. Mathijssen NM, Verburg H, van Leeuwen CC, Molenaar TL, Hannink G (2016) Factors influencing length of hospital stay after primary total knee arthroplasty in a fast-track setting. Knee Surg Sports Traumatol Arthrosc 24(8):2692-2696

6. Schotanus MG, Bemelmans YF, Grimm B, Heyligers IC, Kort NP (2016) Physical activity after outpatient surgery and enhanced recovery for total knee arthroplasty. Knee Surg Sports Traumatol Arthrosc. doi:10.1007/s00167-016-4256-1

7. van Beek R, Zonneveldt HJ, van der Ploeg T, Steens J, Lirk P, Hollmann MW (2017) In patients undergoing fast track total knee arthroplasty, addition of buprenorphine to a femoral nerve block has no clinical advantage: a prospective, double-blinded, randomized, placebo controlled trial. Medicine 96(27):e7393 